\title{
Teste de condutividade elétrica para sementes de pinhão-manso (Jatropha curcas L. $)^{1}$
}

\author{
Electrical conductivity test for physic nut (Jatropha curcas L.) \\ Roberto F. Araujo, João B. Zonta ${ }^{2 *}$,Eduardo F. Araujo ${ }^{3}$, \\ Sérgio M. L. Donzeles ${ }^{2}$, Gabriel M. Costa ${ }^{3}$
}

\section{RESUMO}

Para uma análise mais completa da qualidade de sementes, faz-se necessário complementar as informações fornecidas pelo teste de germinação com testes de vigor, possibilitando, assim, selecionar os melhores lotes para comercialização e semeadura. Dentre esses testes, destaca-se o de condutividade elétrica. Para sementes de pinhão-manso (Jatropha curcas L.), não há informações referentes à metodologia do teste de condutividade elétrica na literatura. Objetivou-se neste trabalho estabelecer metodologia para o teste de condutividade elétrica em sementes de pinhão-manso, variando-se o número de sementes, a quantidade de água e o período de embebição. Para tanto, foram utilizados oito lotes de sementes de pinhão-manso, e o teste de condutividade elétrica foi realizado com as seguintes variações: 15,25 ou 35 sementes, embebidas em 75 ou $100 \mathrm{~mL}$ de água destilada à $25^{\circ} \mathrm{C}$, sendo as leituras realizadas após 3, 6, 9, 12, e 24 horas de embebição. Para efeito de comparação, foram determinadas a porcentagem de germinação e a porcentagem e índice de velocidade de emergência. Com base nos resultados obtidos e pelos coeficientes de correlação calculados entre os resultados de condutividade elétrica e porcentagem de emergência, verificou-se que as metodologias mais eficientes foram com 15 sementes e $75 \mathrm{~mL}$ de água, com leituras após 6 horas, 9 horas e 12 horas e 25 sementes e $75 \mathrm{~mL}$ de água, com leituras, também, após 6 horas, 9 horas e 12 horas. Concluiu-se que o teste de condutividade elétrica foi eficiente para diferenciar lotes de pinhão-manso quanto à qualidade fisiológica das sementes. A metodologia mais adequada para a realização do teste de condutividade elétrica para sementes de pinhão-manso é a conduzida com o uso de 15 sementes e $75 \mathrm{~mL}$ de água, com leitura após 6 horas de embebição.

Palavras chave: Condutividade elétrica, pinhão-manso, vigor, sementes.

\begin{abstract}
In order to have more accurate information of seed quality among different seed lots, it is necessary to supplement the data supplied by the germination test with other tests, making possible the selection of best lots for marketing and seeding establishment and among those tests the electrical conductivity test fits well to that purpose. For physic nut (Jatropha curcas L.), no information about electrical conductivity test methods is available in the literature. Thus, the aim of this research was to establish a standard method for testing the electrical conductivity of physic nut seeds leaking, testing different number of seeds, amount of water and soaking period. So, we used eight lots of physic nut seeds proceeding the electrical conductivity test with the following variations: 15,25 or 35 seeds, soaked in 75 or $100 \mathrm{~mL}$ of distilled water at $25^{\circ} \mathrm{C}$, and the readings performed after 3, 6, 9, 12, and 24 hours of soaking. As a matter of fact, were determined the germination percentage and the seedling emergence rate. Based on the results and on the correlation coefficients calculated between the results of electrical conductivity test and emergence percentage, the most efficient methods were with 15 seeds under soaking in $75 \mathrm{~mL}$ of water, with readings after 6, 9 and 12 hours; 25 seeds under soaking in $75 \mathrm{~mL}$ of water, with readings at the same time mentioned before, but the most appropriate method to to distinguish physic nut seed lots with different physiological quality is the use of 15 seeds in $75 \mathrm{~mL}$ of water, with reading after six hours of its starting.

Key words: Electric conductivity test, physic nut, vigor, seed.
\end{abstract}

1 Trabalho financiado pela FAPEMIG.

2 Departamento de Fitotecnia/Universidade Federal de Viçosa, 36570-000, Viçosa-MG, fone: (0XX31) 38991138, E-mail: jobazonta@hotmail.com, efaraujo@ufv.com, gabrielmoraescosta@yahoo.com.br

* Autor para correspondência. 


\section{Introdução}

Motivado pela oscilação do preço do petróleo, questões ambientais e sociais, nos últimos anos, intensificaram-se as buscas por combustíveis alternativos e renováveis para a substituição parcial ou total dos combustíveis fósseis. Dentre as possibilidades estudadas, o biodiesel tem lugar de destaque. Além do caráter renovável, aspectos sociais impulsionam as pesquisas e implantações de unidades produtoras por todo mundo.

O Brasil tem potencial para se tornar um dos maiores produtores de biodiesel do mundo por dispor de solo e clima adequados ao cultivo de oleaginosas. Há dezenas de espécies vegetais no Brasil das quais se pode extrair o biodiesel, tais como mamona, dendê (palma), girassol, babaçu, amendoim e soja (Castro et al., 2005).

O pinhão-manso (Jatropha curcas L.) é uma nova cultura que vem sendo estudada como alternativa para regiões quentes e secas do país. Para dar suporte técnico ao desenvolvimento da cultura do pinhão-manso, estudos em diferentes áreas devem ser realizados, principalmente os relacionados com produção, colheita, secagem, armazenamento e qualidade de sementes, insumo fundamental para o sucesso de qualquer atividade agrícola. Vale destacar que, para as nossas condições, são escassas as informações na literatura científica sobre produção e tecnologia de sementes dessas oleaginosas, principalmente, de pinhão-manso.

A utilização de qualquer espécie não tradicional, para plantios com finalidade produtiva e/ou ambiental, requer o desenvolvimento de tecnologia adequada de produção, iniciando pelo conhecimento da qualidade da semente.

Como acontece para todas as culturas, a utilização de sementes de boa qualidade é fundamental para o estabelecimento de populações adequadas em campo. Para uma análise mais completa da qualidade de sementes, faz-se necessário complementar as informações fornecidas pelo teste de germinação com testes de vigor, possibilitando, assim, selecionar os melhores lotes para comercialização e semeadura. Dentre esses testes, destaca-se o de condutividade elétrica. Este teste baseia-se na permeabilidade das membranas, avaliando características relacionadas à liberação de metabólitos durante a embebição das sementes (Matthews \& Powell, 1981; AOSA, 1983; Marcos-Filho et al., 1987; Barros \& MarcosFilho, 1997).
Este teste é internacionalmente aceito e recomendado para ser usado em sementes de ervilha pela International Seed Testing Association (Matthews $\&$ Powell, 1981) e para sementes de ervilha e soja pela Association of Official Seed Analysts (AOSA, 1983; Hampton \& Tekrony, 1995). No Brasil, é utilizado como teste de vigor com resultados bastante promissores, principalmente para sementes de milho (Vieira et al., 1995; Fagioli, 1997) e soja (Marcos-Filho et al., 1982; Marcos-Filho et al., 1990; Vieira, 1994; Paiva-Aguerro, 1995; Dias \& Marcos-Filho, 1996).

A pesquisa tem demonstrado que vários fatores podem afetar os resultados do teste, tais como: idade da semente, genótipo, qualidade da água, temperatura e duração do período de embebição, grau de umidade e número de sementes (Vieira, 1994).

Em relação ao período de embebição, Krzyzanowski et al. (1991) e Vieira \& Krzyzanoski (1999) citam que para espécies de sementes grandes, como ervilha e soja, o período de embebição é em torno de 24 horas, pois durante este período ainda há lixiviação. Em sementes pequenas, como as de hortaliças, a lixiviação máxima pode ocorrer num período inferior a duas horas (Murphy \& Noland, 1982).

Para o número de sementes da amostra existem várias recomendações e Loeffler et al. (1988) propõe a utilização de quatro repetições de 50 sementes para a realização do teste de condutividade elétrica, como forma de reduzir o coeficiente de variação (Vieira $\&$ Krzyzanowski, 1999). Alguns trabalhos, visando adequar o teste a determinada espécie, avaliaram diferentes números de sementes. Ribeiro et al. (1997) concluíram que para sementes de milho foi possível detectar diferenças na qualidade fisiológica dos lotes em ambas as temperaturas e tamanho das amostras testadas; entretanto, as repetições de 25 sementes apresentaram melhores resultados do que as de 50 sementes. Rodo et al. (1998) observaram em tomate que o teste de condutividade elétrica foi mais eficiente quando foram utilizadas 50 sementes para a cultivar IAC e com 25 sementes para a cultivar Kada. Contudo, Sá (1999) observou que o tamanho da amostra (25, 50 e 100 sementes) não afeta os valores de condutividade elétrica para as cultivares de tomate Petomech e Santa Clara.

Para sementes de pinhão-manso, não há informações referentes à metodologia do teste de condutividade elétrica na literatura. Assim, objetivouse, no presente trabalho, estabelecer metodologia 
para o teste de condutividade elétrica em sementes de pinhão-manso, variando-se o número de sementes, a quantidade de água e o período de embebição.

\section{Material e Métodos}

O experimento foi conduzido no Laboratório da EPAMIG Zona da Mata e no Laboratório de Sementes do Departamento de Fitotecnia, da Universidade Federal de Viçosa, em Viçosa-MG.

Após a colheita, os frutos de pinhão-manso foram secados à sombra e descascados à mão. Em seguida, as sementes foram submetidas a uma secagem final, à sombra, até o teor de água de $10 \%$. Foram obtidos oito lotes de sementes, provenientes do beneficiamento e classificação em peso e tamanho.

Portanto, obtiveram-se oito lotes de sementes, caracterizados, a seguir, e foram utilizados para testar as diferentes metodologias do teste de condutividade elétrica: Lote 1 = sementes pesadas não classificadas em peneira (PNC); Lote $2=$ sementes pesadas e grandes $(\mathrm{PG})$; Lote $3=$ sementes pesadas e médias $(\mathrm{PM})$; Lote $4=$ sementes pesadas e pequenas (PP); Lote $5=$ sementes leves não classificadas em peneira (LNC); Lote $6=$ sementes leves e grandes (LG); Lote 7 = sementes leves e médias (LM); Lote $8=$ sementes leves e pequenas (LP).

Para a caracterização física e fisiológica das sementes, os lotes foram submetidos às seguintes avaliações:

1. Determinação do Teor de água: a determinação foi realizada utilizando-se o método da estufa, a $105 \pm 3{ }^{\circ} \mathrm{C}$, durante 24 horas, com duas subamostras, conforme especificações das Regras para Análise de Sementes (Brasil, 1992), sendo os resultados expressos em porcentagem.

2. Teste de germinação: oito repetições de 25 sementes foram semeadas em rolo de papel germitest (três folhas), umedecidos com água destilada na proporção de 2.5 vezes seu peso seco. Após a montagem, os rolos foram mantidos em germinador, na temperatura constante de $30{ }^{\circ} \mathrm{C}$. A avaliação foi feita no quinto e décimo dias após a montagem e o resultado expresso em percentagem de plântulas normais;

3. Primeira contagem do teste de germinação: realizada conjuntamente com o teste de germinação e consistiu do registro da percentagem de plântulas normais obtida no quinto dias após a montagem do teste.
4. Teste de emergência: foram utilizadas quatro repetições de 50 sementes, semeadas em leito de areia. Após 10 dias realizou-se a contagem do número de plântulas emergidas e o resultado foi expresso em percentagem.

5. Índice de velocidade de emergência (IVE): foram utilizadas quatro repetições de 50 sementes, semeadas em leito de areia. A cada dia, a partir da emergência da primeira plântula, foram realizadas contagens do número de plântulas emergidas, até que o valor permanecesse constante. Para o cálculo do IVE foi utilizada a fórmula:

$\mathrm{IVE}=\mathrm{E}_{1} / \mathrm{N}_{1}+\mathrm{E}_{2} / \mathrm{N}_{2}+\ldots+\mathrm{E}_{\mathrm{n}} / \mathrm{N}_{\mathrm{n}}$, em que: $\mathrm{IVE}=$ Índice de velocidade de emergência, $\mathrm{E}_{1}, \mathrm{E}_{2}, \mathrm{E}_{\mathrm{n}}=$ número de plântulas emergidas na primeira, na segunda e na última contagem; $\mathrm{N}_{1}, \mathrm{~N}_{2}, \mathrm{~N}_{\mathrm{n}}=$ número de dias da semeadura à primeira, à segunda e à última contagem (Maguire, 1962).

O teste de condutividade elétrica em estudo foi realizado com quatro repetições de 15,25 ou 35 sementes, pesadas e acondicionadas em copos plásticos descartáveis, com capacidade para $200 \mathrm{~mL}$, contendo $75 \mathrm{ou} 100 \mathrm{~mL}$ de água destilada. Em seguida, o material foi levado para BOD, regulada a $25^{\circ} \mathrm{C}$, e a leitura foi realizada após 3, 6, 9, 12 e 24 horas de embebição. A leitura da condutividade foi realizada em condutivímetro e os resultados calculados em $\mu \mathrm{S} \mathrm{cm} \mathrm{cm}^{-1} \mathrm{~g}^{-1}$ (Vieira \& Krzyzanowski, 1999).

Foi utilizado o delineamento inteiramente casualizado, isoladamente para cada quantidade de sementes e quantidade de água, com a comparação de médias pelo teste de Tukey (5\% de probabilidade). Para cada metodologia estudada, foi calculado o coeficiente de correlação de Pearson entre os resultados obtidos na leitura do teste de condutividade elétrica e a porcentagem de emergência em areia, sendo a significância dos valores de correlação determinada pelo teste $\mathrm{t}$ a $1 \%$ de probabilidade (Gomes, 2000).

\section{Resultados e Discussão}

Os resultados referentes ao teor de água das sementes (Tabela 1) foram semelhantes para os oito lotes estudados. Esse fato é importante para a execução dos testes, pois deve haver uniformidade do teor de água inicial das sementes para obtenção de resultados consistentes (Loeffler et al., 1988). Verificou-se que o teor de água dos lotes situou-se 
Tabela 1. Teor de água (TA), germinação (G), primeira contagem (PC), emergência (E) e índice de velocidade de emergência (IVE) de sementes de pinhão-manso com diferentes pesos específicos e tamanhos.

\begin{tabular}{|c|c|c|c|c|c|}
\hline LOTES & TA (\%b.u.) & $\mathrm{G}(\%)$ & $\mathrm{PC}$ & $\mathrm{E}(\%)$ & IVE \\
\hline PNC & 8.2 & $98 \mathrm{a}$ & $95 \mathrm{a}$ & $100 \mathrm{a}$ & $8.3 \mathrm{a}$ \\
\hline PG & 8.1 & $99 a$ & $96 a$ & $99 \mathrm{a}$ & $7.9 \mathrm{a}$ \\
\hline PM & 8.3 & $98 \mathrm{a}$ & $93 a$ & $100 \mathrm{a}$ & $7.2 \mathrm{a}$ \\
\hline PP & 8.3 & $98 \mathrm{a}$ & $95 \mathrm{a}$ & $98 \mathrm{a}$ & $6.9 \mathrm{a}$ \\
\hline LNC & 8.2 & $76 b$ & $58 \mathrm{~b}$ & $72 b$ & $4.4 \mathrm{~b}$ \\
\hline LG & 8.1 & $70 \mathrm{~b}$ & $54 \mathrm{~b}$ & $72 \mathrm{~b}$ & $4.3 \mathrm{~b}$ \\
\hline LM & 8.0 & $74 \mathrm{~b}$ & $59 \mathrm{~b}$ & $78 \mathrm{~b}$ & $4.3 \mathrm{~b}$ \\
\hline LP & 8.3 & $66 \mathrm{c}$ & $57 \mathrm{~b}$ & $76 b$ & $4.4 \mathrm{~b}$ \\
\hline $\mathrm{CV}(\%)$ & - & 11.58 & 13.36 & 7.83 & 15.36 \\
\hline
\end{tabular}

1 Médias seguidas pela mesma letra na coluna não diferem entre si pelo teste de Skott-Knott a 5\% de probabilidade.

Tabela 2. Condutividade elétrica $\left(\mu \mathrm{S} . \mathrm{cm}^{-1} \cdot \mathrm{g}^{-1}\right)$ de oito lotes de sementes de pinhão manso, utilizando-se 15 sementes embebidas em 75 e $100 \mathrm{~mL}$ de água destilada durante $3,6,9,12$ e 24 horas a $25^{\circ} \mathrm{C}$.

\begin{tabular}{|c|c|c|c|c|c|}
\hline \multicolumn{6}{|c|}{ Embebição em 75 mL de água destilada } \\
\hline \multirow{2}{*}{ LOTES } & \multicolumn{5}{|c|}{ Período de embebição (horas) } \\
\hline & 3 & 6 & 9 & 12 & 24 \\
\hline PNC & $91.329 b^{1}$ & $109.793 \mathrm{~b}$ & $117.856 \mathrm{~b}$ & $123.067 \mathrm{~b}$ & $130.391 \mathrm{~b}$ \\
\hline PG & $113.147 \mathrm{~b}$ & $121.542 \mathrm{~b}$ & $132.974 \mathrm{~b}$ & $138.295 \mathrm{~b}$ & $169.643 \mathrm{a}$ \\
\hline PM & $111.371 \mathrm{~b}$ & $123.946 \mathrm{~b}$ & $132.257 \mathrm{~b}$ & $141.153 \mathrm{~b}$ & $149.400 \mathrm{~b}$ \\
\hline PP & $124.869 \mathrm{a}$ & $133.410 \mathrm{~b}$ & $144.299 \mathrm{~b}$ & $151.345 \mathrm{~b}$ & $160.967 \mathrm{a}$ \\
\hline LNC & $138.605 \mathrm{a}$ & $154.215 \mathrm{a}$ & $164.068 \mathrm{a}$ & $179.452 \mathrm{a}$ & $190.349 \mathrm{a}$ \\
\hline LG & $133.929 \mathrm{a}$ & $151.527 \mathrm{a}$ & $160.601 \mathrm{a}$ & $170.424 \mathrm{a}$ & $186.418 \mathrm{a}$ \\
\hline LM & $133.699 \mathrm{a}$ & $147.004 \mathrm{a}$ & $161.188 \mathrm{a}$ & $165.828 \mathrm{a}$ & $171.897 \mathrm{a}$ \\
\hline LP & $130.821 \mathrm{a}$ & $144.554 \mathrm{a}$ & $158.718 \mathrm{a}$ & $160.073 \mathrm{a}$ & $175.880 \mathrm{a}$ \\
\hline $\mathrm{CV}(\%)$ & 11.44 & 8.75 & 9.01 & 10.02 & 11.90 \\
\hline \multicolumn{6}{|c|}{ Embebição em 100 mL de água destilada } \\
\hline $\mathrm{PNC}$ & $76.556 \mathrm{~b}$ & $87.779 \mathrm{~b}$ & $93.069 \mathrm{c}$ & $95.550 \mathrm{~b}$ & $109.105 \mathrm{~b}$ \\
\hline PG & $72.388 \mathrm{~b}$ & $82.187 \mathrm{~b}$ & $85.726 \mathrm{c}$ & $87.767 \mathrm{c}$ & $103.248 \mathrm{~b}$ \\
\hline PM & $75.309 \mathrm{~b}$ & $88.746 \mathrm{~b}$ & $93.201 \mathrm{c}$ & $96.937 \mathrm{~b}$ & $112.656 \mathrm{~b}$ \\
\hline PP & $80.025 \mathrm{~b}$ & $94.546 \mathrm{a}$ & $98.706 \mathrm{~b}$ & $102.410 \mathrm{a}$ & $119.421 \mathrm{~b}$ \\
\hline LNC & $80.467 \mathrm{~b}$ & $95.396 \mathrm{a}$ & $100.822 \mathrm{~b}$ & $108.031 \mathrm{a}$ & $131.049 \mathrm{a}$ \\
\hline LG & $79.779 \mathrm{~b}$ & $96.081 \mathrm{a}$ & $101.515 \mathrm{~b}$ & $105.607 \mathrm{a}$ & $130.737 \mathrm{a}$ \\
\hline LM & $86.138 \mathrm{a}$ & $100.845 \mathrm{a}$ & $107.957 \mathrm{a}$ & $111.618 \mathrm{a}$ & $129.090 \mathrm{a}$ \\
\hline LP & $88.225 \mathrm{a}$ & $105.209 \mathrm{a}$ & $111.143 \mathrm{a}$ & $112.563 \mathrm{a}$ & $134.973 \mathrm{a}$ \\
\hline $\mathrm{CV}(\%)$ & 6.96 & 6.43 & 5.98 & 5.08 & 5.77 \\
\hline
\end{tabular}

1 Médias seguidas pela mesma letra na coluna não diferem entre si pelo teste de Skott-Knott a 5\% de probabilidade.

em torno de 8,0 a $8,3 \%$, ou seja, dentro da faixa que não influenciaria no teste de condutividade, de acordo com Vieira \& Krzyzanowski (1999).

Pela análise geral dos resultados dos testes de germinação, de primeira contagem da germinação, de emergência e do índice de velocidade de emergência (Tabela 1), observa-se que a classificação dos lotes de sementes de pinhão-manso, em relação ao seu potencial fisiológico, não variou em função do teste utilizado. É possível destacar o desempenho superior dos quatro primeiros lotes, compostos por sementes mais pesadas, em relação aos quatro lotes restantes, compostos por sementes mais leves. Os lotes com sementes mais leves apresentaram potencial fisiológico inferior em todos os testes realizados.

Pelos resultados do teste de condutividade elétrica, utilizando-se 15 sementes e $75 \mathrm{~mL}$ de água (Tabela 2) destaca-se também a superioridade da qualidade das sementes mais pesadas (quatro primeiros 
lotes) em relação à qualidade das sementes mais leves (quatro últimos lotes) nas leituras realizadas após 6, 9 e 12 horas de embebição das sementes. Quando a leitura foi realizada após os períodos de 3 e 24 horas de embebição, a diferenciação da qualidade fisiológica dos lotes não teve comportamento semelhante à dos demais testes de qualidade (Tabela 1). Quando se utilizaram 15 sementes e $100 \mathrm{~mL}$ de água (Tabela 2), as leituras realizadas, após todos os períodos de embebição, com exceção da leitura após 24 horas, não possibilitaram a caracterização dos lotes com a mesma eficiência que a metodologia em que se utilizaram 15 sementes e $75 \mathrm{~mL}$ de água, nas leituras realizadas após 6, 9 e 12 horas de embebição das sementes.

Quando o teste de condutividade elétrica foi realizado com 25 sementes e $75 \mathrm{~mL}$ de água (Tabela 3), independente do período de embebição, também houve a diferenciação dos lotes de maneira semelhante à metodologia em que se utilizaram 15 sementes e $75 \mathrm{~mL}$ de água, nos tempos de embebição de 6 horas em diante.

Para as demais metodologias (25 sementes e $100 \mathrm{~mL}$ de água, 35 sementes e $75 \mathrm{~mL}$ de água e 35 sementes e $100 \mathrm{~mL}$ de água) (Tabelas 3 e 4), a diferenciação dos lotes não foi tão eficiente quanto às metodologias em que se utilizaram 15 ou 25 sementes e $75 \mathrm{~mL}$ de água, com leituras realizadas após 6, 9 e 12 horas de embebição de sementes, que estratificaram os oito lotes de maneira semelhante aos demais testes de vigor utilizados (Tabela 1).

Para melhor explicação dos resultados, foi calculado o coeficiente de correlação de Pearson entre todos os tratamentos estudados no teste de condutividade elétrica e os valores do teste de emergência (Tabela 5). Com base nos resultados obtidos pelo coeficiente de correlação, pode-se verificar que as melhores metodologias foram com 15 sementes e $75 \mathrm{~mL}$ de água, com leituras após 6 horas $(\mathrm{r}=-0.910), 9$ horas $(\mathrm{r}=-0.880)$ e 12 horas $(\mathrm{r}=-0.920)$ e 25 sementes e $75 \mathrm{~mL}$ de água, com leituras, também, após 6 horas $(\mathrm{r}=-0.818), 9$ horas $(\mathrm{r}=-0.853)$ e 12 horas $(\mathrm{r}=-0.865)$. Como um dos objetivos dos testes de laboratório é o de reduzir tempo e material e, considerando-se os testes de média, os coeficientes de correlação, a rapidez para obtenção dos resultados, o tempo de uso da BOD e a economia de material, a metodologia mais recomendada é o uso de 15 sementes e $75 \mathrm{~mL}$ de água, com leitura após 6 horas de embebição.

Tabela 3. Condutividade elétrica $\left(\mu \mathrm{S} . \mathrm{cm}^{-1} \cdot \mathrm{g}^{-1}\right)$ de oito lotes de sementes de pinhão manso, utilizando-se 25 sementes embebidas em 75 e $100 \mathrm{~mL}$ de água destilada durante $3,6,9,12$ e 24 horas a $25^{\circ} \mathrm{C}$.

\begin{tabular}{|c|c|c|c|c|c|}
\hline \multicolumn{6}{|c|}{ Embebição em 75 mL de água destilada } \\
\hline \multirow{2}{*}{ LOTES } & \multicolumn{5}{|c|}{ Período de embebição (horas) } \\
\hline & 3 & 6 & 9 & 12 & 24 \\
\hline PNC & $104.188 b^{1}$ & $112.001 \mathrm{~b}$ & $122.222 \mathrm{~b}$ & $129.790 \mathrm{~b}$ & $140.257 \mathrm{~b}$ \\
\hline PG & $105.691 \mathrm{~b}$ & $111.818 \mathrm{~b}$ & $122.239 \mathrm{~b}$ & $127.985 \mathrm{~b}$ & $139.641 \mathrm{~b}$ \\
\hline PM & $110.845 \mathrm{~b}$ & $120.025 \mathrm{~b}$ & $130.208 \mathrm{~b}$ & $138.094 \mathrm{~b}$ & $147.938 \mathrm{~b}$ \\
\hline PP & $105.272 \mathrm{~b}$ & $113.844 \mathrm{~b}$ & $123.047 \mathrm{~b}$ & $130.135 \mathrm{~b}$ & $144.236 \mathrm{~b}$ \\
\hline LNC & $114.772 \mathrm{a}$ & $128.296 \mathrm{a}$ & $143.619 \mathrm{a}$ & $150.467 \mathrm{a}$ & $164.912 \mathrm{a}$ \\
\hline LG & $125.071 \mathrm{a}$ & $137.166 \mathrm{a}$ & $149.954 \mathrm{a}$ & $157.617 \mathrm{a}$ & $179.755 \mathrm{a}$ \\
\hline LM & $121.928 \mathrm{a}$ & $133.681 \mathrm{a}$ & $149.632 \mathrm{a}$ & $153.724 \mathrm{a}$ & $158.878 \mathrm{a}$ \\
\hline LP & $119.794 \mathrm{a}$ & $132.681 \mathrm{a}$ & $145.007 \mathrm{a}$ & $149.179 \mathrm{a}$ & $173.248 \mathrm{a}$ \\
\hline $\mathrm{CV}(\%)$ & 7.68 & 6.72 & 7.08 & 6.50 & 7.65 \\
\hline \multicolumn{6}{|c|}{ Embebição em 100 mL de água destilada } \\
\hline PNC & $68.322 \mathrm{~b}$ & $78.877 \mathrm{~b}$ & $83.125 \mathrm{~b}$ & $84.361 \mathrm{c}$ & $101.383 \mathrm{~b}$ \\
\hline PG & $67.022 \mathrm{~b}$ & $77.233 \mathrm{~b}$ & $79.769 \mathrm{~b}$ & $81.999 \mathrm{c}$ & $100.190 \mathrm{~b}$ \\
\hline PM & $70.700 \mathrm{~b}$ & $80.772 \mathrm{~b}$ & $84.698 \mathrm{~b}$ & $88.165 \mathrm{~b}$ & $108.222 \mathrm{~b}$ \\
\hline $\mathrm{PP}$ & $72.741 \mathrm{~b}$ & $82.799 \mathrm{~b}$ & $88.120 \mathrm{~b}$ & $92.232 \mathrm{~b}$ & $110.014 \mathrm{~b}$ \\
\hline LNC & $74.025 \mathrm{~b}$ & $86.991 \mathrm{~b}$ & $90.243 \mathrm{~b}$ & $93.949 \mathrm{~b}$ & $117.012 \mathrm{a}$ \\
\hline LG & $81.663 \mathrm{a}$ & $96.163 \mathrm{a}$ & $101.067 \mathrm{a}$ & $105.649 \mathrm{a}$ & $131.443 \mathrm{a}$ \\
\hline LM & $81.366 \mathrm{a}$ & $93.197 \mathrm{a}$ & $98.533 \mathrm{a}$ & $102.199 \mathrm{a}$ & $126.177 \mathrm{a}$ \\
\hline LP & $70.083 \mathrm{~b}$ & $83.252 \mathrm{~b}$ & $87.470 \mathrm{~b}$ & $91.232 \mathrm{~b}$ & $117.334 \mathrm{a}$ \\
\hline CV (\%) & 5.67 & 6.02 & 6.23 & 5.93 & 7.82 \\
\hline
\end{tabular}

1 Médias seguidas pela mesma letra na coluna não diferem entre si pelo teste de Skott-Knott a 5\% de probabilidade. 
Tabela 4. Condutividade elétrica $\left(\mu \mathrm{S} . \mathrm{cm}^{-1} \cdot \mathrm{g}^{-1}\right)$ de oito lotes de sementes de pinhão manso, utilizando-se 35 sementes embebidas em 75 e $100 \mathrm{~mL}$ de água destilada durante $3,6,9,12$ e 24 horas a $25^{\circ} \mathrm{C}$.

\begin{tabular}{|c|c|c|c|c|c|}
\hline \multicolumn{6}{|c|}{ Embebição em 75 mL de água destilada } \\
\hline \multirow{2}{*}{ LOTES } & \multicolumn{5}{|c|}{ Período de embebição (horas) } \\
\hline & 3 & 6 & 9 & 12 & 24 \\
\hline $\mathrm{PNC}$ & $99.636 b^{1}$ & $105.812 \mathrm{~b}$ & $118.708 \mathrm{~b}$ & $124.270 \mathrm{~b}$ & $135.637 \mathrm{~b}$ \\
\hline PG & $98.181 \mathrm{~b}$ & $105.298 \mathrm{~b}$ & $114.062 \mathrm{~b}$ & $122.418 \mathrm{~b}$ & $136.853 \mathrm{~b}$ \\
\hline PM & $110.756 \mathrm{a}$ & $117.787 \mathrm{a}$ & $127.947 \mathrm{~b}$ & $135.316 \mathrm{~b}$ & $150.131 \mathrm{~b}$ \\
\hline $\mathrm{PP}$ & $114.773 \mathrm{a}$ & $124.781 \mathrm{a}$ & $133.859 \mathrm{a}$ & $142.556 \mathrm{a}$ & $158.940 \mathrm{a}$ \\
\hline LNC & $120.044 \mathrm{a}$ & $132.311 \mathrm{a}$ & $142.358 \mathrm{a}$ & $152.800 \mathrm{a}$ & $168.876 \mathrm{a}$ \\
\hline LG & $114.281 \mathrm{a}$ & $125.945 \mathrm{a}$ & $142.982 \mathrm{a}$ & $147.045 \mathrm{a}$ & $163.398 \mathrm{a}$ \\
\hline LM & $115.152 \mathrm{a}$ & $130.187 \mathrm{a}$ & $141.676 \mathrm{a}$ & $150.667 \mathrm{a}$ & $165.534 \mathrm{a}$ \\
\hline LP & $106.952 \mathrm{~b}$ & $119.810 \mathrm{a}$ & $134.021 \mathrm{a}$ & $142.202 \mathrm{a}$ & $162.958 \mathrm{a}$ \\
\hline $\mathrm{CV}(\%)$ & 8.49 & 8.27 & 8.09 & 7.68 & 8.16 \\
\hline \multicolumn{6}{|c|}{ Embebição em 100 mL de água destilada } \\
\hline PNC & $62.477 \mathrm{~b}$ & $70.706 \mathrm{c}$ & $75.200 \mathrm{~b}$ & $77.328 \mathrm{~b}$ & $94.242 \mathrm{c}$ \\
\hline PG & $61.532 \mathrm{~b}$ & $68.501 \mathrm{c}$ & $73.816 \mathrm{~b}$ & $78.409 \mathrm{~b}$ & $97.113 \mathrm{c}$ \\
\hline PM & $66.797 \mathrm{~b}$ & $76.729 \mathrm{~b}$ & $81.144 \mathrm{~b}$ & $84.905 \mathrm{~b}$ & $105.145 \mathrm{~b}$ \\
\hline $\mathrm{PP}$ & $71.482 \mathrm{a}$ & $80.023 \mathrm{~b}$ & $85.715 \mathrm{a}$ & $90.577 \mathrm{a}$ & $110.148 \mathrm{~b}$ \\
\hline LNC & $74.535 \mathrm{a}$ & $88.504 \mathrm{a}$ & $92.786 \mathrm{a}$ & $98.686 \mathrm{a}$ & $125.376 \mathrm{a}$ \\
\hline LG & $77.769 \mathrm{a}$ & $92.447 \mathrm{a}$ & $98.068 \mathrm{a}$ & $102.895 \mathrm{a}$ & $133.787 \mathrm{a}$ \\
\hline LM & $72.530 \mathrm{a}$ & $86.922 \mathrm{a}$ & $91.416 \mathrm{a}$ & $96.215 \mathrm{a}$ & $120.602 \mathrm{a}$ \\
\hline LP & $74.351 \mathrm{a}$ & $87.376 \mathrm{a}$ & $94.133 \mathrm{a}$ & $97.777 \mathrm{a}$ & $121.864 \mathrm{a}$ \\
\hline $\mathrm{CV}(\%)$ & 6.80 & 6.79 & 6.70 & 6.45 & 6.83 \\
\hline
\end{tabular}

1 Médias seguidas pela mesma letra na coluna não diferem entre si pelo teste de Skott-Knott a 5\% de probabilidade.

Tabela 5. Coeficiente de correlação de Pearson entre os valores do teste de condutividade elétrica, para todos os tratamentos, e os valores do teste de emergência em areia, de oito lotes de sementes de pinhão manso.

\begin{tabular}{|c|c|c|c|c|c|}
\hline & \multicolumn{5}{|c|}{ Condutividade elétrica } \\
\hline & \multicolumn{5}{|c|}{ Período de embebição (horas) } \\
\hline & 3 & 6 & 9 & 12 & 24 \\
\hline Emergência em areia & \multicolumn{5}{|c|}{15 sementes $75 \mathrm{~mL}$ de água destilada } \\
\hline Emergência em areia & \multicolumn{5}{|c|}{15 sementes $100 \mathrm{~mL}$ de água destilada } \\
\hline Emergência em areia & \multicolumn{5}{|c|}{25 sementes $75 \mathrm{~mL}$ de água destilada } \\
\hline \multirow[t]{2}{*}{ Emergência em areia } & \multicolumn{5}{|c|}{25 sementes $100 \mathrm{~mL}$ de água destilada } \\
\hline & \multicolumn{5}{|c|}{35 sementes $75 \mathrm{~mL}$ de água destilada } \\
\hline Emergência em areia & $-0.674^{* *}$ & $-0.753 * *$ & $-0.810^{* *}$ & $-0.810 * *$ & $-0.806^{* *}$ \\
\hline Emergência em areia & $-0.813^{* *}$ & $\begin{array}{r}35 \mathrm{se} \\
-0.864^{* * *}\end{array}$ & $\begin{array}{c}100 \mathrm{~mL} \text { de á } \\
-0.843^{* *}\end{array}$ & $\begin{array}{l}\text { lada } \\
-0.861 * *\end{array}$ & $-0.887 * *$ \\
\hline
\end{tabular}

** Significativo a $1 \%$ de probabilidade pelo teste $\mathrm{t}$.

Em relação ao período de embebição, no presente trabalho a classificação dos lotes foi mais consistente após o período de 6 horas de embebição, utilizando-se 100 sementes por repetição, o que representa uma significativa redução no período de condicionamento das sementes em relação ao período de 24 horas, adotado pela pesquisa como padrão para testes de condutividade elétrica para soja (Vieira \& Krzyzanowski, 1999). Resultados semelhantes foram observados com sementes de feijão-de-vagem e de quiabo (Dias et al., 1998), de tomate (Rodo et al., 1998), de brócolos (Martins et al., 2002), de berinjela (Novembre et al., 2002) e de amendoim (Vanzolini e Nakagawa, 1999ab). 


\section{Conclusões}

- O teste de condutividade elétrica foi eficiente para diferenciar lotes de pinhão-manso quanto à qualidade fisiológica das sementes;

- Recomenda-se a utilização de 15 sementes e $75 \mathrm{~mL}$ de água, com leitura após 6 horas de embebição.
Agradecimento: À Fundação de Amparo à Pesquisa do Estado de Minas Gerais (FAPEMIG), pelo patrocínio desse projeto de pesquisa e pela concessão da bolsa de pesquisa ao primeiro autor.

\section{Referências Citadas}

AOSA. Association of official seed analysts

1983 Seed vigor testing handbook. East Lasing, 93 p. (Contribution, 32).

BRASIL, Ministério da Agricultura e Reforma Agrária. Regras para análise de sementes.

1992 Brasília: DNDV/SNAD/CLAV, 365 p.

Barros, A.S.R.; Marcos-Filho, J.

1997 Testes para avaliação rápida do vigor de sementes de soja. Revista Brasileira de Sementes, v. 19, n. 2, pp. 289295

Castro, C.E.F.; Carbonell, S.A.M.; Maia, M.S.D.; Morais, C.G 2005 Biodiesel. 1. ed. Campinas, 62 p. (CONSEPA, SÉRIE REUNIÕES TÉCNICAS).

Dias, D.C.F.S.; Marcos-Filho, J.

1996 Testes de condutividade elétrica para avaliação do vigor de sementes de soja (Glycine $\max (\mathrm{L}$.) Merrill). Scientia Agricola, v. 53, n. 1, pp. 31-42.

Dias, D.C.F.S.; Vieira, A.N.; Bhering, M.C.

1998 Condutividade elétrica e lixiviação de potássio para avaliação do vigor de sementes de hortaliças: feijão-devagem e quiabo. Revista Brasileira de Sementes, v. 20, n. 2 , pp. 408-413.

Fagioli, M.

1997 Relação entre a condutividade elétrica de sementes e a emergência das plântulas de milho em campo. Dissertação Mestrado, 74f. Faculdade de Ciências Agrárias e Veterinárias/ UNESP, Jaboticabal, SP.

Gomes, F.P.

2000 Curso de estatística experimental. 14 ed., PiracicabaSP, $477 \mathrm{p}$.

Hampton, J.G.; Tekrony,B.M.

1995 Conductivity test. In: Hampton, J.G.; Tekrony, B.M. (eds.) Handbook of vigour methods. 3.ed. Zürich: ISTA, pp. 22-34.

Krzyzanowski, F.C.; França Neto, J.B.; Henning, A.A. 1991 Relato dos testes de vigor disponíveis para as grandes culturas. Informativo ABRATES, v. 1, n. 2, pp. 15-37.

Loeffler, T.M.; Tekrony, D.M.; Egli, D.B. 1988 The bulk conductivity test as na indicator of soybean seed quality. Journal Seed Technology, v. 12, n. 1, pp. 37-53.

Maguirre, J.D.

1962 Speed of germination-aid in selection and evaluation for seedling emergence and vigour. Cropp. Science, Madison, v. 2, n. 2, pp. 176-177.

Marcos-Filho, J.; Cícero, S.M.; Silva, W.R.

1987 Avaliação da qualidade das sementes. Piracicaba: FEALQ, 230 p.
Marcos-Filho, J.; Amorin, H.V.; Silvarolla, M.B.; Pescarin, H.M.C.

1982 Relações entre germinação, vigor e permeabilidade das membranas celulares durante a maturação de sementes de soja. In: Seminário Nacional de Pesquisa de Soja, 2, Brasília, 1981. Anais... Londrina: EMBRAPA-CNPSo, v. 1, pp. 676-688.

Marcos-Filho, J.; Silva, W.R.; Novembre, A.D.C.; Chamma, H.M.C.P.

1990 Estudo comparativo de métodos para ênfase ao teste de condutividade elétrica. Pesquisa Agropecuária Brasileira, v. 25, n. 12, pp. 1805-1815.

Martins, C.C.; Martinelli-Seneme, A.; Castro, M.M.; Nakagawa, J.; Cavariani, C.

2002 Comparação entre métodos para a avaliação do vigor de lotes de sementes de couve-brócolos (Brassica oleracea L. var. italica Plenk). Revista Brasileira de Sementes, v. 24, n. 2, pp. 96-101.

Matthews, S.; Powell, A.A.

1981 Electrical condutivity test. In: Perry, D.A. (ed.). Handbook of vigour methods. Zürich: ISTA, pp. 37-42.

Murphy, J.B.; Noland, T.L.

1982 Temperature effects on seed imbibition and leakage mediated by viscosity and membranes. Plant Physiology, v. 69 , n. 2 , pp. $428-431$.

Novembre, A.D.L.C.; Carpi, V.A.F.; Marcos Filho, J.; Chamma, H.M.C.P.

2002 Teste de condutividade elétrica para estimar o potencial fisiológico de sementes de berinjela. Horticultura Brasileira, v. 20, n. 2, 293 p.

Paiva-Aguerro, J.A.

1995 Correlação de condutividade elétrica e outros testes de vigor com a emergência das plântulas de soja em campo. Dissertação Mestrado, 74f. Faculdade de Ciências Agrárias e Veterinárias/UNESP, Jaboticabal, SP.

Ribeiro, D.M.C.A.; Carvalho, M.L.M.; Salgado, K.C.C.

1997 Avaliação da qualidade fisiológica de sementes de milho através do teste de condutividade elétrica (bulk). Informativo Abrates, v. 7, n. 1/2, p. 187.

Rodo, A.B.; Tillmann, M.A.A.; Villela, F.A.; Sampaio, N.V. 1998 Teste de condutividade elétrica em sementes de tomate. Revista Brasileira de Sementes, v. 20, n. 1, pp. 29-38.

Sá, M.E.

1999 Condutividade elétrica em sementes de tomate (Lycopersicon lycopersicum L.). Scientia Agricola, v. 56, n. 1, pp. 13-19. 
Vanzolini, S.; Nakagawa, J.

1999a Teste de condutividade elétrica em sementes de amendoim: efeitos de temperatura e de período de embebição. Revista Brasileira de Sementes, v. 21, n. 1, pp. 41-45.

Vanzolini, S.; Nakagawa, J.

1999b Teste de condutividade elétrica em sementes de amendoim: efeitos de teor de água inicial e de período de embebição. Revista Brasileira de Sementes, v. 21, n. 1, pp. 46-52.

Vieira, R.D.; Minohara, L.; Carvalho, N.M.; Bergamaschi, M.C.M.
1995 Relationship. of black layer and milk line development to maize seed maturity. Scientia Agricola, v. 52, n. 1, pp. 142147.

Vieira, R.D.

1994 Teste de condutividade elétrica. In: Vieira, R.D.; Carvalho, N.M. (ed.). Testes de vigor em sementes. Jaboticabal: FUNEP, pp. 103-132.

Vieira, R.D.; Krzyzanowski, F.C.

1999 Teste de condutividade elétrica. In: Krzyzanowski, F.C. Vieira, R.D.; França-Neto, J.B. (Ed.). Vigor de sementes: conceitos e testes. Londrina: ABRATES, cap. 4, pp. 1-26. 